\title{
SOME REMARKS ON POISSON TYPE INTEGRALS IN THE POLYBALL
}

\author{
R.F. SHAMOYAN
}

\author{
Date of Receiving : $\quad 31.08 .2019$ \\ Date of Revision : $\quad 31.12 .2019$ \\ Date of Acceptance : 17.02 .2020
}

\begin{abstract}
We consider some natural extensions of Poisson integral in the unit ball to polyballs and extend some known classical results to the case of product domains(polyballs). In particular we extend some known results in the unit ball on Poisson integrals related to BMO to the product domain case
\end{abstract}

\section{Introduction}

Let $B_{n}=|z|<1$ be the unit ball in $C^{n}, S^{n}=\partial B$ be the unit sphere in $C^{n}$

Let $d(z, w)=|1-<z, w>|^{\frac{1}{2}}, z, w \in \bar{B}_{n}$, be the restriction of $d$ on $S_{n}$.It is $a$ non isotropic metric. (see $[1,2]$ ).

Let also $Q(\xi, r)=\left\{\eta \in S_{n}:|1-<\xi, \eta>|^{\frac{1}{2}}<r\right\}, r>0, \xi \in S_{n}$. We call $Q$ d-ball, $S_{n}=\{|z|=1\}$. We denote various constants in this paper by $C, C_{1}, C_{2}$. We define Poisson kernel $P(z, w), z, w \in \bar{B}_{n}, P(z, w)=\frac{\left(1-|z|^{2}\right)^{n}}{|1-<\bar{z}, w>|^{2 n}}$; and a Poisson integral of a positive Borel measure $\mu, P(\mu)$ in a standard way.(see,for example, [1]).We refer to [1] and [3-6] for some new and classical results on these objects.

In this note we discuss some problems in open new research area of Poisson type integrals in product domains in $\mathbb{C}^{n}$. Some new objects in this note will be defined and some interesting new problems will be also posed and solved.We provide first Unit ball known results. (see[1]).

In $[1,2]$ it was shown that

$$
\int_{S_{n}}|f(\eta)-f(a)|^{2} P(a, \eta) d \sigma(\eta) \geq\left(\frac{\tilde{c}}{\sigma(Q)} \int_{Q}|f-f(a)|^{2} d \sigma\right) ;
$$

2010 Mathematics Subject Classification. 47B33, 30H99.

Key words and phrases. Poisson integral, unit ball, d-balls, Carleson type measures, polyball. Communicated by: Shiv.K.Kaushik 